\title{
A Review: Embedding a Simulation Training Program in Labor and Delivery to Improve Healthcare Quality, Clinical Outcomes, and Patient Satisfaction
}

\author{
Dana K. Bryant \\ New York City Health and Hospitals/Kings County Hospital, New York, USA \\ Email: Dana.Bryant@nychhc.org
}

How to cite this paper: Bryant, D.K. (2019) A Review: Embedding a Simulation Training Program in Labor and Delivery to Improve Healthcare Quality, Clinical Outcomes, and Patient Satisfaction. Open Journal of Obstetrics and Gynecology, 9, 12261249.

https://doi.org/10.4236/ojog.2019.99120

Received: July 18, 2019

Accepted: September 7, 2019

Published: September 10, 2019

Copyright $\odot 2019$ by author(s) and Scientific Research Publishing Inc. This work is licensed under the Creative Commons Attribution International License (CC BY 4.0).

http://creativecommons.org/licenses/by/4.0/

\begin{abstract}
In response to maternal deaths being three-fold higher for black women than other races in New York City, Governor Andrew M. Cuomo created a taskforce which met for a year. In March 2019, the taskforce published ten recommendations to address the current healthcare disparities. The taskforce recommended utilizing medical simulation training to improve skills and communication. Peer-reviewed literature was explored for evidentiary support for embedding a simulation program in a NYC public hospital. In addition, obstacles to and advantages of in-situ simulation programs were discussed. Steps for garnering executive leadership support, improving teamwork, defining outcome measures, and identifying beneficiaries of in-situ simulation programs were explored.
\end{abstract}

\section{Keywords}

Embedded Simulation, Postpartum Hemorrhage Drills, Interdisciplinary

Teaching, Teamwork in Obstetrics, Qualitative and Quantitative

Improvements in Obstetrical Outcomes

\section{Introduction}

Black non-Latina women in New York City (NYC) have the highest Severe Maternal Morbidity (SMM) rates in the country [1]. This disparity has led to a city and state wide effort to reform obstetrical care. The majority of poor health outcomes in the healthcare industry are related to poor communication and poor teamwork [2]. Medical simulation has been proven to improve communication, 
teamwork, quality, and patient outcomes [3]. However, many institutions do not effectively utilize medical simulation training. This review explores the multiple issues that a healthcare institution must address in order to embed a simulation program within a Labor and Delivery unit (L \& D).

\subsection{Historical Perspective}

Historically the practice of medicine consisted of the solo practitioner with the "doctor knows best" attitude. "People were cared for by one all-knowing doctor who lived in the community, visited the home, and was available to attend to needs at any time of day or night" [4]. Subsequently medical institutions developed systems and processes in silos and personal preference was the primary consideration when decisions were made.

Currently, medicine is practiced with multidisciplinary teams where the doctor usually leads the team. Evidence-based practice has replaced physicians' experience. Standardization compliments the physicians' personal preference.

In 2000, the Institute of Medicine (IOM) released a book To Err is Human: Building a Safer Health System. This book addressed the fact that at least 44,000, and perhaps as many as 98,000 patients die in hospitals each year from medical errors [2]. In response to this IOM report "the Clinton administration issued an executive order instructing a government agency that conducts or oversees health care programs. The intention of the agency was to implement proven techniques for reducing medical errors. Additionally, the president creating a task force to find new strategies for reducing errors" [2]. In December 2000 congress appropriated 50 million dollars to the Agency for Healthcare Research and Quality (AHRQ) to support efforts to reduce medical errors. Part of the AHRQ action plan was developing and testing new technologies to reduce medical errors [5]. This prompted the medical industry to seriously re-consider simulation training. "A significant amount of research evidence supports the benefits of simulation" [5].

Simulation is an embedded part of training in the airline industry. The first flight simulator was used for training in 1910. (https://www.proflight.com) [6] "Commercial pilots are currently required to undergo simulation training every 6 months. As a result, aviation accidents have been significantly reduced. Between 2004 and present no major US carrier had an aircraft accident which resulted in loss of life" [7]. Boeing very recently had two planes crash; one taking off from Jakarta, Indonesia, and the other from Addis Ababa, Ethiopia. There is worldwide focus on airplane safety. One could argue that the healthcare industry should be held to higher standards than the airline industry.

Simulation prevents actual patients from being the provider's "first experience" and it allows medical staff to practice events that are rare. Simulations can be filmed so that participants can observe themselves in a variety of clinical scenarios and hear their responses to questions. In addition, it allows providers to receive feedback about their clinical performance. In the entertain- 
ment industry professionals would never perform without rehearsing. One could argue that in a critical industry like medicine, providers should not "perform" without rehearsing.

\subsection{Obstetric Emergencies and Simulation}

Postpartum hemorrhage (PPH) continues to be one of the leading causes of maternal morbidity and mortality in the world. "Hemorrhage that leads to blood transfusion is the leading cause of severe maternal morbidity in the United States" [8]. It has been recommended by some that a hemorrhage response team should be organized to manage this obstetric emergency [9]. L and D units have implemented a PPH code where all available L and D employees are involved along with hospital transporters, an administrator, and a representative from the internal medicine department.

Medical simulation drills that allow employees to practice obstetric emergencies like PPH are prudent. In addition to individual training, simulation can be used to help "work teams". "Work teams" are continuing units responsible for producing goods or providing services. Their membership is typically stable, usually full-time and well defined [10]. Hospital units depend on "work teams" for optimal functioning. However, there are many barriers that can inhibit effective teamwork. These barriers are: "lack of mutual trust, inability to manage conflict, lack of clarity in instructions, poorly defined roles and responsibilities, and finally a negative atmosphere" [10]. Simulations and the subsequent debriefing sessions directly address all of these barriers.

\subsection{New York City Statistics}

In 2016 the New York city department of health published statistics that revealed that black women experience three times the rate of poor pregnancy outcomes when compared to women of other races [1]. Brooklyn has had the highest rates of SMM and maternal mortality in NYC. From 2008-2012, East Flatbush had an SMM rate of 500 per 10,000 deliveries. The rate among all New York City residents during this same time-frame was 231.9 per 10,000 deliveries [1]. The current maternal morbidity and mortality statistics in NYC compel hospitals that serve predominately black patient populations to embed simulation training as one way to improve patient outcomes.

\subsection{New York State Taskforce}

In response to the report from the New York City Department of Health, Governor Andrew M. Cuomo created a taskforce. This taskforce first met in April of 2018 and was "part of a multi-pronged effort to reduce maternal mortality and racial disparities. The taskforce met three times between June and December 2018" (www.governor.ny.gov). This focus group created a document that describes ten "top taskforce recommendations". Two of the ten recommendations were directly related to simulation programs and training. Recommendation num- 
ber seven was "create competency-based curricula for providers as well as medical and nursing schools" and recommendation number ten was "promote universal birth preparedness and postpartum continuity of care" (www.governor.ny.gov). In addition to the 'top taskforce recommendations' this report highlights the opinion that the Governor must support efforts that "enhance authentic learning opportunities with multidisciplinary teams, such as simulation, to improve skills and communication with pre-/post-assessment to determine outcomes" (www.governor.ny.gov) [11].

\subsection{Theoretical Framework}

Neilssen, et al., 2017 conducted a study involving 89 healthcare workers in Tanzania.The workers underwent simulation training. Immediately after the simulation training the workers took a multiple-choice test. Nine months after the first test, the 89 healthcare workers were given a second exam. Both examinations were designed to test the workers knowledge about postpartum hemorrhage. The researchers concluded that there was a need for continued training because knowledge and skills deteriorated after nine months.

This study [12] supported the idea that simulation drills need to be repeated so that healthcare workers can retain their skills and knowledge. In addition, the Neilssen study suggested ways in which the needs of a unit can be assessed and then effectively addressed by the simulation trainer. This paper is a foundation for future investigations to determine the frequency with which simulation drills should occur.

Dahlberg, Nelson, Dahlgren, \& Blomberg [13] conducted a study in Sweden in which a simulation-based training program was introduced on a labor and delivery unit. "Data concerning maternal characteristics, management, and obstetric outcomes was compared between three groups" [13]. The groups were pre-simulation training (2004-2007), immediately after simulation training began (2008-2011), and after simulation training had been implemented for many years (2012-2015).

The results of this study [13] revealed that implementing the shoulder dystocia training program led to a significant decrease in injuries related to delivery. Brachial plexus injury can result from shoulder dystocia. The pre-drill rate for brachial plexus injury was $73 \%$ and the late post-simulation rate was $17 \%$. This decrease in delivery related injury was significant at a p-value of greater than 0.05 .

Both of these studies provide evidentiary support that utilizing simulation programs can lead to improved patient outcomes. These studies evaluated two of the most devastating obstetric emergencies that are encountered on a daily basis throughout labor and delivery units worldwide. Both studies emphasize the importance of continued training and tracking outcome data. The studies provide a framework for both creating a training/drilling schedule and for tracking and recording outcome data. Creating a simulation program that includes all obstetric emergencies is logical. 


\subsection{Research Questions}

The questions posed in this review are related to implementation and outcome metrics. They are as follows: What is required for embedding a simulation program in a labor and delivery unit?; What costs/resources must be considered when implementing a simulation program?; and What are the anticipated positive outcomes once the program is implemented?

\subsection{Qualitative Research Methods}

The peer-reviewed literature provided evidentiary support for all aspects of this simulation review. Allocating the money, resources, and workforce needed to implement a simulation program is prudent if it will improve patient outcomes. Literature was reviewed qualitatively to determine if simulation training leads to increased quality of healthcare. In addition, the review of literature addressed costs, stakeholder buy-in, and most importantly outcome measures for mother and baby. A formal meta-analysis with quantitative data is forthcoming.

\subsection{Professional Experience}

Clinical experience in obstetrics repeatedly demonstrates the value of teamwork. The lesson is that effective teamwork saves patients' lives. Obstetric emergencies are unpredictable and can be catastrophic. Effective communication and teamwork do not always happen naturally particularly in high stress situations. A well-managed obstetric emergency is one where a "situation leader" manages the emergency by managing the time, assigning roles, and alerting the team as to their progress. For example, during a shoulder dystocia the baby needs to be delivered in under five minutes or irreversible infant harm can occur.

In emergency situations many healthcare providers look to the most senior person in the room to be the situation leader. Sometimes the most senior person is not the best person to lead the team. Professional experience has taught all of us that, in healthcare, when all team members are valued for their unique strengths and abilities, teams can function optimally. In obstetrics, seconds can make the difference between good patient outcomes and patient harm for both mother and baby. Effective teamwork saves lives and improves patient outcomes.

The history of simulation training in high-reliability industries is well established. The need for improvement in communication and teamwork in healthcare is evidenced by the unacceptably high number of preventable deaths. Current healthcare disparities and poor obstetric outcomes make labor and delivery units an ideal setting for embedding simulation training programs. It is imperative that a hospital that serves an at-risk community utilizes every available tool to improve quality and patient outcomes. Simulation training is an example of such a training tool. In addition, it is an ethical responsibility for NYC public hospitals to directly address racial disparities in healthcare.

Gaining support for the resources required to embed a simulation program is critical. Executive leadership in healthcare organizations will be compelled to 
support a project that is cost effective, improves patient satisfaction, promotes employee engagement, and produces improvement in outcome metrics that can be measured. Increasing the quality of healthcare without increasing healthcare costs is a mandate for healthcare executives. The next section will review evidence supporting the need for an embedded simulation program within the healthcare institution. Literature regarding the resources required, costs incurred, and the return on investment will be discussed. Literature that discusses the obstacles to and advantages of simulation training are explored.

\section{Literature Review}

Embedding a simulation program in a labor and delivery unit requires both buy-in from all stakeholders and financial support. Evidence-based research that demonstrated the effectiveness of simulation training, as well as its associated costs, are reviewed. The need for simulation is further supported by demographic information about the healthcare institution's patient population. In addition, literature regarding obstacles to and advantages of simulation training were reviewed.

\subsection{Institutional Need}

A review of the 2008-2012 executive summary from the Severe Maternal Morbidity (SMM) report published by the New York City Department of Health highlighted the need for simulation. New York City (NYC) public hospitals serve the most vulnerable populations within city borders. "New York City's rate of SMM was 1.6 times the national rate from 2008-2009, and Black non-Latina women had the highest SMM rate-three times that of White non-Latina women. The rate remained high even after stratifying by other known risk factors such as low education level, neighborhood poverty level, and pre-pregnancy obesity" [1].

The author's professional experience working in a NYC public city hospital has revealed that there are hospitals that serve patient populations that are over 95\% percent black. These hospitals have a moral obligation to utilize all available teaching and training tools that are known to improve: teamwork, quality of care, and patient outcomes.

\subsection{Clinical Care and Simulation}

A research study published in 2015 by Al-Dahlwali, Airan, Elsherif, Tawfeeq, \& Mokhele was designed to help clinicians improve clinical accuracy when estimating blood loss during infant delivery. One-hundred twenty-three health care workers in Saudi Arabia were involved in this study from January to June in 2011. "The participants were subject to three research phases and an educational intervention" [14]. Initially all participants underestimated the amount of blood loss. The training and education improved the participants' accuracy at a statistically significant level ( $\mathrm{p}$-value $<0.0001$ ). Postpartum hemorrhage is one of the leading causes of maternal morbidity in the world and in NYC. It is logical to 
assume that this demonstrable improvement in accuracy will lead to an improvement in patient outcome secondary to more accurate utilization of blood transfusions.

Fritz, Walker, Cohen, Angeles, and Lamadrid-Figueroa [15] conducted a randomized control trial of an intervention that was designed to measure "the impact of a simulation and team-training program (PRONTO) on the performance of evidence-based practices in normal births". The number of participants was large enough to demonstrate the effectiveness of simulation training. For example, when evaluating an obstetric emergency (shoulder dystocia) there was a 22 percent increase in the correct management for a $\mathrm{p}$-value of 0.001 . The research design used in this study, a randomized control trial, is considered the "goldstandard" of clinical research. This research provided strong support that simulation can improve the clinical skills of providers of obstetric care.

Mittal [16] addressed simulation training for surgeons. The author discussed evidence that simulation training improved surgical skills "such as skin suturing, knot tying, airway management, peripheral IV placement, nasogastric tube placement, Foley catheterization, and central line placement" [16]. It is important to remember that obstetricians and gynecologists are surgeons and require a similar set of surgical skills.

\subsection{Costs Related to Simulation}

Embedding a simulation program within a healthcare institution will incur costs.

Convincing executive leadership that money and other resources should be utilized for such a project is critical. The costs include, but are not limited to, the following: equipment, information technology, the maintenance of the physical space used for training, costs associated with teaching, simulation trainers, and indirect costs like the loss of direct patient care revenue hours.

In 2017 Bukari, Andreatta, Goldiez, \& Rabelo published a journal article that described a framework that healthcare organizations can use to determine the costs and benefits of simulation training [17]. The framework was based on three respected methodologies; one of which is utilized by several departments of the U.S. government to determine return on investments.

Organizations can evaluate both quantitative and qualitative costs and benefits in terms of "real dollars" by utilizing this described method. This framework can be utilized to calculate the costs associated with simulation training in a "dollar amount". We hope to use their methodology in our future work.

Costs related to medical malpractice must also be considered. A perfect baby and mother are the expected outcome when a woman gives birth. If a different outcome occurs the result is often a lawsuit for medical negligence. The costs associated with medical lawsuits are direct and indirect. New York City paid 123 million dollars for" medical malpractice claims at its public hospitals" [18]. A significant portion was paid for obstetric-related outcomes. The indirect costs are more life-changing: loss of a provider's confidence, depression among all labor 
and delivery personnel, and increased stress. These are the substance of burnout and it is well known that burnout can lead to dysfunctional behavior at work.

Many studies have revealed that good communication is an effective way for healthcare providers to mitigate lawsuits. For example, a peer reviewed paper entitled Apologies and Medical Error stated the following: "Focus groups of patients indicated that patients desire and expect to be informed promptly about a medical error; to be given information about what occurred, why and how it occurred, how their health will be affected, and what steps will be taken to prevent future harm; and to receive an apology that signals a sense of regret and a desire to do better going forward". A complete review of this literature is forthcoming. Experimental studies provide evidence that apologies may serve to facilitate settlement of claims." [19].

Simulation is a tool that many healthcare institutions utilize to teach providers how to apologize to patients. The Association of American Medical Colleges acknowledges that communication is a core competency that is often taught by using filmed activities followed by debriefing sessions to teach medical students' effective ways of communicating apologies and medical errors to patients. Decreasing the financial and emotional toll of medical lawsuits can significantly decrease costs to healthcare organizations [5].

\subsection{Obstacles to Simulation Programs}

There are many obstacles that must be confronted when an institution begins to embed simulation training into its departments. For example, employee burnout, the organizational culture, and poor planning can hinder the success of any simulation program.

\subsection{Employee Engagement}

Recognizing employee burnout and implementing corrective actions is an important step in the success of any simulation program. "Employee burnout is a progressive psychological response to chronic work stress involving emotional exhaustion, depersonalization, and feelings of reduced personal accomplishment" [20]. The National Institute of Standards and Technology (NIST) published the 2019-2020 Baldrige Excellence Framework. This publication provides information on the Baldrige framework which empowers an organization to reach its goals, improve results, and become more competitive. "The framework consists of the Criteria, the core values, and concepts, and the scoring guidelines for those applying for a Baldrige award" (www.nist.gov). A core value of the Baldrige framework is workforce engagement. This manual stated that "the success of your workforce members-including your leaders-depends on their having opportunities to learn. This learning includes preparing people for future organizational core competencies" (www.nist.gov). It is imperative that leadership describe simulation as a learning opportunity. Participating in simulation training allows employees to learn new techniques and utilize clinical expertise. 
Allowing time for training and allocating resources for simulation will reinforce the idea that leadership values the workforce and wants to provide proven ways of supporting employees' skills.

\subsection{Organizational Culture}

Weiner [21] published an article that examined organizational readiness for change. "The theory described in this article treats organizational readiness as a shared psychological state in which organizational members feel committed to implementing an organizational change and confident in their collective abilities to do so". The article explored the conditions that promote change, change efficacy, contextual factors, and organizational outcomes.

Implementing a simulation training program in a labor and delivery unit requires change throughout the organization. Protocols, procedures, and the allocation of money are some changes that will occur within the organization. An understanding of factors that promote as well as hinder organizational change will help when implementing the simulation program.

In addition to readiness for change, the overall organizational culture may need to be addressed prior to embedding a simulation program. For example, The Greater New York Hospital Association [22] published a resource guide for healthcare providers. This guide discussed the importance of the principle of a "just culture" organization. Just culture is an organizational "culture that seeks to balance the need to learn from mistakes with the need to hold staff accountable" (www.gnyha.org). Healthcare leadership "need to create an environment in which staff are comfortable reporting errors and near-misses without threat of blame and retribution" (www.gnyha.org). If the organizational culture is plagued by blaming and threats then simulation drills will not be as effective. A simulation manual published in 2016 explained that the simulation team must feel safe. Feeling safe is defined as simulation participants being "comfortable participating, speaking up, sharing thoughts, and asking for help as needed without concern for retribution or embarrassment and that the team is safe for risk taking, and mistakes will be considered learning opportunities rather than there being embarrassment or punitive consequences" (www.healthandospital.org). This feeling of safety during simulation drills cannot exist in an organization plagued by blaming, retaliation, and fear of reporting near-misses.

\subsection{Project Planning}

Good projects often fail because of poor planning. The Harvard Business Review Guide to Project Management [23] described four phases of project management.

These four phases are: "planning, build-up, implementation, and closeout" [23]. Planning involves identifying stakeholders, defining project objectives, and defining tasks. In addition, identifying a change agent to lead the project is important. Anderson [24] described 5 roles that change agents may play during the planning phase of any project. These roles are as follow: "1) Facilitative: Helping 
a client or group attain its desired outcomes by clarifying alternatives, processes, and decisions. 2) Gatekeeping: Acting as a boundary-spanner between groups or between a supervisor and a team, negotiating between them and giving objective feedback. 3) Diagnostic: Pointing out what has been seen, heard, or learned to enhance group or individual awareness. 4) Architectural: Designing situations, events, and conversations so that awareness, learning, and change can occur. 5). Mobilizing: Advocating for a particular approach or perspective" [24].

The build-up phase involves assembling the team, developing assignments, organizing meetings, developing a budget, and providing a list of deliverables. Many healthcare leaders are formally trained in and have expertise in project planning. However, many leaders admit to having great difficulty when attempting to implement projects or change existing programs. The 4 Disciplines of Execution is a book is an in-depth guide for "strategic organizational change". This book is highly respected and has received worldwide recognition from CEOs of Fortune 500 companies and other industry leaders. Embedding a simulation program requires both planning and execution. The 4 Disciplines of $E_{X-}$ ecution [25] provides techniques and examples of how program implementation has been achieved in successful companies. The book directly addressed the most problematic issues related to program implementation and provides solutions that have been proven to work. The lessons learned and shared by the leaders quoted in this book are applicable to the obstetric simulation project.

\subsection{Advantages of Simulation Programs}

Medical simulation offers many advantages to healthcare organizations. Simulation drills are a way that test current systems and processes. In addition, simulation improves teamwork, and patient outcomes.

\subsection{Systems and Processes}

Personal professional experiences support the fact that simulation exposes weaknesses within hospital systems and processes. For example, during an Advanced Cardiac Life Support (ACLS) drill, held during residency training, it was discovered that a few hospital beds were broken. The headboard was unable to be removed and this inhibited the anesthesiologist when simulating intubation. If this occurred during a real cardiac arrest it would be considered a preventable medical error.

A second experience with simulation training revealed the fact that the overhead announcement equipment was not able to be heard in a critical are within the hospital. In addition, the audiovisual staff was not appropriately trained to understand when, why, and how certain emergency codes were to be announced.

\subsection{Teamwork}

It has been accepted as fact by multiple regulatory agencies like the Agency for Healthcare Research and Quality (AHRQ) and Joint Commission that teamwork 
and communication are needed to provide high quality healthcare. "The high-performance team is now widely recognized as an essential tool for constructing a more patient-centered, coordinated, and effective health delivery system." [4].

O'Leary, Sehgal, Terrell, \&Williams published an article that addressed communication of interdisciplinary teams in hospitals. "A Joint Commission study indicated that communication failures were the root cause for two-thirds of the 3548 sentinel events reported from 1995 to 2005”. In addition to communication this article discussed the physical location of the physicians within the hospital, daily goals and checklists, interdisciplinary rounds, and teamwork training. The authors recommend "the implementation of a combination of interventions with adaptations to fit unique clinical settings and local culture". A simulation program that is embedded within a labor and delivery unit has the ability to address the authors' recommendations for improvement in interdisciplinary teamwork.

\subsection{Patient Outcomes}

A study protocol was published by Banga et al. in 2014 [26]. The protocol was implemented in the Netherlands. The Netherlands had a slightly higher rate of poor obstetric outcomes than other European countries. Dutch auditors concluded that the obstetric collaborative in the Netherlands could do a better job with decreasing maternal morbidity and mortality. The researchers implemented simulation-based training in obstetric units. The belief was that the training would improve team performance and patient outcomes. This study protocol outlined several strategies for teaching team skills during simulation. In addition, the researchers defined the learning objectives and outcome measures. This information will be valuable when creating the curriculum and defining the outcome measures for the simulation program that is being created. The patient outcomes being studied in this research project are like those that are collected by the NYC Department of Health and Mental Hygiene SMM project. The results of this study are yet to be published.

This chapter provided peer-reviewed literature that addressed numerous aspects of medical simulation training and program implementation. Evidence of effectiveness, associated costs, organizational considerations, and outcome measures were considered. The next section will provide a discussion regarding implementation of a simulation program. The Baldrige framework will be considered throughout this review. The experience of established programs, obtaining executive support, teamwork, change theory and change management will be discussed.

\section{Discussion and Analysis}

There are medical simulation centers located throughout the country. Jacobi Hospital, located in the Bronx borough of NYC, has a multimillion dollar "state of the art" simulation center. Jacobi's simulation center hosts a myriad of educa- 
tional sessions and simulation sessions throughout every year. Despite the availability of this simulation center it is recommended that New York city hospitals embed simulation programs within their organizations. Potential advantages to embedding simulation programs are as follows: they allow the work flow to be analyzed, they can lead to improvement in organizational culture, they provide opportunities to practice teamwork and communication skills, care teams can practice rare events more frequently, and simulation can identify problems within the systems and processes in the hospital [5].

\subsection{Executive Support}

The first step for embedding a simulation program within a unit is organizing the core team. The core team should include representatives from different stake-holder groups. The stakeholders in this simulation project are as follows: a trained simulation instructor, labor and deliver nurses and physicians, a unit clerk, housekeeping, hospital police, hospital transport, blood bank personnel, anesthesiologists, pediatricians, internists, resident physicians, and midwives.

After the core team is identified the next step is ensuring the support of organizational executive leadership. Executive leadership has the power to give financial support, allocate materials and resources, and provide physical space. In addition, confirming support from the departmental chairman, "medical and nursing leadership, allied health leadership, risk management and patient safety" is advisable (www.nychealthandhospitals,org/simulationcenter) The systems and processes within a particular hospital may also require the buy-in from other hospital areas like hospital police, administrative clerks, blood bank, environmental services, material management, laboratory, admitting, and information technology.

Executive leadership in healthcare is required to justify expenditures. The U.S. has one of the most expensive healthcare industries in the world but does not have comparably better outcomes than countries that spend less on healthcare [27]. Therefore, if the simulation core-team can present a budget that is fiscally responsible and outcomes measures that support increased quality then garnering executive support will be more likely. Including the previously mentioned framework described by Bukari, Andreatta, Goldiez, and Rabelo [17] is important. This will allow for intangible benefits and qualitative benefits to be outlined and monetized. Secondly, defining and explaining meaningful outcome measures will secure buy-in. This can be challenging in obstetrics because "current quality indicators may not be sufficiently comprehensive for guiding quality improvement in obstetric care". Despite this fact, it is imperative that healthcare organizations continue to work towards defining meaningful outcome measures.

Executive support of organizational programs is a hallmark of Baldrige award winning hospitals. The best hospitals have leaders that take intelligent risks, implement innovations, and support both performance improvement and produc- 
tivity (www.nist.gov/baldrige, 2019). Adopting the Baldrige framework for healthcare initiatives is logical [28].

\subsection{Teamwork}

Leaders in healthcare are addressing quality and patient safety by utilizing work teams. The goal is for high-performance work teams to become the norm within the healthcare industry. "A high-performance work team refers to a group of goal-focused individuals with specialized expertise and complementary skills who collaborate, innovate, and produce consistently superior results"

(https://www.shrm.org, 2015) [10]. Medical simulation is used to improve interdisciplinary teamwork and promote superior performance. "A healthcare system that supports effective teamwork can improve the quality of patient care, enhance patient safety, and reduce workload issues that cause burnout among healthcare professionals" (www.chsrf.ca) [29].

It is prudent for the simulation core-team to present peer-reviewed research that demonstrates the effectiveness of simulation training and the need for on-going training. Evidence will garner both philosophical and financial executive support. In addition, an adaptation of these types of studies can be developed and used to measure teamwork goals that are specific to the organization's $\mathrm{L}$ and $\mathrm{D}$ unit. The results of the $\mathrm{L}$ and $\mathrm{D}$ unit's prospective qualitative study can be analyzed using one of the algorithms described above to monetize the effectiveness of the simulation program. This type of plan will compel executive leadership and promote buy-in from stakeholders.

Monod, Voekt, Gisin, Gisin, and Hoesli [30] conducted a study that investigated the influence of simulation training on four specific skills:" self-confidence, handling of emergency situations, knowledge of algorithms and team communication". One hundred sixty-eight participants took part in the simulation training. Three months after training, the 156 participants returned Likert scale questionnaires that revealed a statistically significant increase in improvement in skills regarding in all four areas. The statements that revealed the improvement areas are as follows: (Before the training,) "I felt helpless". (After the training I realized) "I had the emergency under control during the scenarios. I had the impression that the communication within the team improved. My way of communication in emergency situations improved". This is an example of a study that can be used to provide evidence to leadership and stakeholders regarding the usefulness of simulation programs.

\subsection{Change Theory/Change Management}

Kurt Lewin is a considered to be one of the founders of what is currently known as change management. The basic premise of Lewin's change theory is that organizations must undergo three steps: 1) unfreezing, 2) change, and 3) refreezing. Unfreezing is the stage where organizational needs, problems, and interventions are defined and the need for change is expressed. The change step is when 
the organization actively implements new protocols, procedures, systems, and programs like simulation. The refreezing step is when the changes are permanently adopted and become what the workforce considers normal practice.

Many consider data gathering to be the most important step in Lewin's "unfreezing" process [23]. In-depth data gathering is important for three reasons: 1) it provides information about the functioning, effectiveness, and vitality of the organization; 2) data can provide an impetus for change; and 3) the act of gathering data can promote positive interpersonal interactions that may not have otherwise occurred. [24]. In addition, gathering data from a variety of sources will provide different perspectives and perceptions about the issue. The data gathering aspect of a simulation project serves multiple purposes. It promotes employee engagement and promotes stakeholder buy-in. Work flow issues, gaps in systems, faulty processes, and outcome measures can be identified prior to implementation of the simulation program.

Organizational development practitioners recommended that an in-depth analysis of the environment be performed. The environment in this instance is the labor and delivery unit. This evaluation is known as a "Strengths, Weaknesses, Opportunities, and Threats (SWOT) analysis and should include data from both quantitative research and personal interviews with key personnel" [31]. The core team for the simulation project can perform this task.

A key part of embedding a simulation program in a labor and delivery unit is strategic planning. Strategic planning helps the core team define the goals and objectives of the unit. Once the critical issues are identified goals can be established. "Each goal needs to be dissected into smaller, more manageable components: Objectives-short term targets in each goal area. Actions-the principal activities that need to be accomplished to achieve the objectives" [32]. In the case of a labor and delivery unit the goal might be to increase the quality of care for patients. An objective could be to decrease the number of infections and hemorrhages. The action is to review all infection and hemorrhage cases that occurred in the previous year and identify areas of weakness. After which, knowledge gaps are addressed, and evidence-based protocols can be implemented to address these two complications. Simulation can be used to address knowledge gaps and teach the workforce the new protocols and procedures that are adopted to decrease the identified complications of infection and hemorrhage.

Even though "developing a good strategic plan is not easy, implementation is far more difficult" [32]. The ability to implement strategies, plans, and protocols is what separates average organizations and units from world class organizations and units. Clear communication is the most important part of implementing and preparing for change.

Memorial Hermann Sugar Land Hospital (MHSL) is a 2017 Baldrige Award recipient. According to the vice president of their Communications and Public Relations Department, Alex Loessin, this organization places communication as one of its "highest priorities" (A, Loessin, personal communication, January 29, 2018). Ms. Loessin explained that a member of the communications team is 
present during the initial phases of all quality improvement and performance improvement projects. They inform both internal and external stakeholders about what is occurring and what to expect in the future. Internally, the communication alerts everyone that a project is underway, and that change is coming. This communication allows for mental preparation to begin prior to procedural changes. External communication alerts the public that MHSL hospital is committed to achieving the best quality and highest performance ratings. The hospital has a reputation for relentlessly pursuing excellence.

Embedding programs within organizations provides many advantages. The advantages include but are not limited to the following: interdisciplinary "work teams" that practice often, knowledge gaps can be identified, process and system errors are exposed and can be corrected, communication and teamwork are enhanced, employee engagement is enhanced, and the quality of patient care is improved.

To ensure proper implementation of a unit embedded simulation program it is important to utilize organizational change management principles." Projects fail at a spectacular rate. One reason is that too many people are reluctant to speak up about their reservations during the all-important planning phase" [23]. Data gathering, SWOT analysis, and ensuring support are all part of the planning phase.

The next section will address sustainability of an embedded simulation program. Securing money, core-team members and their responsibilities, outcome measures, and embedded simulation as it relates to the value proposition equation will be highlighted. Lastly, the impact of an embedded L and D simulation program will be discussed.

\section{Conclusions}

Simulation training in the medical industry is becoming widely accepted as an effective way to teach: communication skills, teamwork, and individual skills. Motola et al. [3] outlined the team competency model of team training. "Team leadership, mutual performance monitoring, backup behavior, adaptability, team orientation, shared mental models, and closed-loop communication" are the outlined competencies. Perfecting these competencies will improve communication and teamwork. "Current research indicates that ineffective communication among health care professionals is one of the leading causes of medical errors and patient harm" [33]. Simulation training is a proven way of improving communication and teamwork. Embedding a simulation training program on a labor and delivery unit is prudent.

\subsection{Sustainability}

After the simulation program is embedded on labor and delivery ensuring its continued success is critical. Martin et al. [34] offered "new insights into the sustainability of organizational change". The study stated, "sustainability requires continuing effort through time, rather than representing a final state to be achieved" [34]. 


\subsection{Core Team}

There must be a simulation team. It is recommended that the team be comprised of members from a variety of areas. The educational personnel are responsible for designing simulation scenarios, leading the simulations, and conducting debriefing sessions. Technical personnel manage the information technology system, the audio-visual equipment, and are responsible for setting up the simulation equipment. Administrative personnel should coordinate the schedule of the simulation participants, track outcome data, and obtain continuing medical education credits when applicable [35].

A "train the trainer" model can be utilized to ensure that there are enough qualified members of the education team to sustain the simulation program. Yarber et al. [36] researched the use of "train the trainer" approaches "for broadly disseminating evidence-based public health principles". The results of this study revealed that $88 \%$ of the study participants agreed that they acquired new knowledge through the "train the trainer "method.

Nestel et al. [37] published a qualitative research article that was developed because of an urgent "need to enhance the quality and scale of simulation-based education" in Australia.

There were multiple training modules and each module had between 45 and 254 respondents. "The content and educational methods were rated highly with items exceeding the pre-established standard". The researchers concluded that "the development of a national training program to support a competent simulation workforce is feasible". Recreating this model on an organizational level is a reasonable goal. On-going "train the trainer "education that is a built-in part of the simulation program will ensure that there is a sustainable, knowledgeable, and qualified workforce available.

\subsection{Funding}

Passiment, Sacks, \& Huang [5] published survey results about medical simulation programs. Survey participants were asked to identify the funding sources for their simulation program. The "financing models included grants/foundation (36\%); revenue generated by courses (32\%), and service to individuals and groups (32\%); for teaching hospital participants. The majority reported that the hospital financed the simulation program (51\%). The study participants were asked questions about their annual budgets.

Included in the budgets were the total full-time equivalent (FTE) personnel for the simulation program. Fifty teaching hospitals responded to this question and the average number of FTE simulation personnel was 3.4 FTE.

Sustainable funding should be considered during the planning phase of the simulation project. Presenting a realistic budget in addition to a projected budget is important when obtaining executive leadership support. To embed a labor and delivery unit-based simulation program successfully requires on-going funding. Long-term success requires presenting peer-reviewed financial information 
and budgets. Executive leadership will have the opportunity to create line item budgets and allocate funds and resources thus ensuring program sustainability.

\subsection{Outcome Measures}

Defining, tracking, and reporting outcome measures is important for ensuring sustainability of the simulation program. The ability to demonstrate improvement in outcome measures promotes stakeholder buy-in, funding, and overall support. Qualitative and quantitative outcome measures should be defined realizing that the measures may change over time. The core team's technology personnel are responsible for tracking this data and compiling reports of the results. It is important to include the technology personnel when outcome measures are being defined because the IT system may require alterations to allow for easier collection and compilation of data.

Outcome metrics may be different depending on the patient population and the specific challenges of individual healthcare organizations. However, there are outcome measures that are applicable in all healthcare organizations and in all specialties in the medicine. These measures can be permanently incorporated into all unit-based simulation programs. An example of a universal simulation outcome metric is situational awareness in medical emergencies. "Nontechnical skills have an impact on health care outcomes and improve patient safety. Situational awareness is core with the view that an understanding of the environment will influence decision-making and performance" [38]. Numerous research papers have defined situation awareness outcome measures and data collection tools. The core team should review these research papers and choose measures and tools that are most appropriate for their individual organization and unit.

\subsection{Impact}

The publication To Err Is Human: Building a Safer Health System [2] firmly established the need for safety and quality improvement initiatives in the healthcare industry. Widespread utilization of medical simulation training programs is a direct result of this report. Unit-based and hospital-based medical simulation has had a positive impact on patients, healthcare providers, and healthcare organizations.

\subsection{Value Proposition}

The equation is "value = quality/cost". This equation is often used to define the "value proposition". Regardless of the industry, value for a consumer equates to the highest quality product or service purchased at the lowest cost" [39]. Unitbased simulation programs both increase quality and decrease cost thus increasing overall value.

\subsection{Quality}

Simulation improves the quality of healthcare by improving teamwork, commu- 
nication, and individual skills. Motola et al. [3] proved that medical simulation improves aspects of teamwork and communication like leadership skills, adaptability, shared mental models, and closed-loop communication. In addition, Motola et al. [3] proved that individual skills like suturing, laparoscopic surgery skills, surgical knot tying, and provider to patient communication skills are improved with participation in a medical simulation training program.

\subsection{Costs}

Simulation training promotes the use of clinical medical guidelines and adherence to medical protocols. [40]. Utilizing evidence-based clinical guidelines and protocols leads to decreased provider variation. "It is well-known throughout the business world that variation and lack of standardization lead to poor quality and higher costs. The same is true in health care, whether the issue is an episode of care around a surgical procedure or long-term management of chronic disease" [41]. In addition, it has been proven that effective communication decreases medical lawsuits which lead to decreased costs.

Litigation in obstetrics is the result of a complex of events when malpractice (presumed or real) impacts on the attitude of pregnant women and their environment. Therefore, to reduce medical-legal issues in obstetrics, we must increase attention to higher standards of obstetric care. This will help to avoid negligence and medical errors and should go along with better communication with pregnant women [42]. Motola et al. proved that effective provider-patient communication with regards to errors and unexpected outcomes is a skill that can be taught with medical simulation [3].

\subsection{Beneficiaries}

Unit-based simulation programs offer a variety of different advantages over simulation centers located in one hospital in a health system. The beneficiaries of an embedded labor and delivery simulation program include providers, patients, and the healthcare organization itself.

\subsection{Healthcare Providers}

Obstetricians, midwives, nurses, resident physicians, ancillary staff, and medical students all benefit from a unit-based simulation program. The benefits include, but are not limited to, the following: opportunity to practice individual skills and teamwork skills; the ability to have impromptu simulation drills; the use of the actual working space; the ability to discover if there are errors in the systems and processes of the unit; decreased out-of-pocket costs for providers; free continuing medical education (CME) credits; and organization-wide involvement when appropriate. Nelissen et al. [12] researched the effect of unit-based simulation training on the labor and delivery staff as it relates to postpartum hemorrhage. They discovered that "simulated performance of basic delivery skills in the management of postpartum hemorrhage" increased significantly and remained 
increased after nine months post-training. Simulation participants demonstrated increased knowledge, skills, and confidence. There are direct benefits of simulation training. However, Nelissen et al. [12] also demonstrated that after nine months skills and knowledge eroded. There is evidence that repeated training is necessary to maintain the benefits of simulation.

\subsection{Patients}

"Patient satisfaction affects clinical outcomes, patient retention, and medical malpractice claims. Patient satisfaction is thus a proxy but a very effective indicator to measure the success of doctors and hospitals" [43]. In addition, patient satisfaction has been shown to increase tremendously when the medical care provider has superior interpersonal skills and communication skills [43]. Medical simulation has been proven to increase providers interpersonal and communication skills [5]. It is intuitive that increased provider knowledge, confidence, and skills will increase the quality of care patients receive. Therefore, simulation programs are beneficial to patients.

\subsection{Healthcare Organizations}

Unit-based simulation programs benefit the organizations in which they are housed. Simulation training has been shown to decrease clinical variation amongst healthcare providers. Decreased clinical variation leads to decreased costs for the organization. In addition, increased provider knowledge, individual skills, and communication leads to increased patient-satisfaction. Increased patient-satisfaction leads to decreased lawsuits and fewer patient complaints. Increased patient-satisfaction leads to a better reputation and increased referrals. Both of which lead to increased revenue for the organization. One of the core values and concepts of "Baldrige Award winning healthcare institutions" is managing innovations. "Innovation means making meaningful change to improve your organization's products, services, programs, processes, operations, and business model, with the purpose of creating new value for stakeholders" (http://www.nist.gov). Embedding a simulation training program on a labor and delivery unit is the type of innovation that separate Baldrige worthy institutions from average healthcare institutions.

\section{Further Conclusions}

In New York City "Black non-Latina women had the highest SMM rate-three times that of White non-Latina women. This rate remains high even after stratifying by other known risk factors such as low education level, neighborhood poverty level and pre-pregnancy obesity". Hospitals that provide obstetric services to predominately black patient populations are obligated to find ways to address this issue. Although other races of women have less SMM events than black women, childbirth continues to remain a high-risk event in women's lives. Medical emergencies related to hemorrhage, hypertension, and blood clotting 
issues cause the majority of poor health outcomes in obstetrics [1].

Embedding simulation training programs in labor and deliver units is one way to address the issues of healthcare disparities and obstetric emergencies. The skills that can be taught in an obstetric simulation program include effective communication skills, surgical skills, and steps for managing obstetric emergencies. Many researchers of medical simulation demonstrated that staff benefited from increased confidence in their skills, patients benefitted because they received higher quality patient-centered care, and organizations benefited by having fewer lawsuits and a better reputation. In addition, the organizational cost savings of a unit based obstetric simulation program comes from decreased variation and increased quality of care. Indirect organizational benefits include an engaged staff, improvement in processes and systems, and improved teamwork.

It is imperative for organizational development principles to be utilized when organizations seek to embed simulation programs within units. The SWOT analysis along with other aspects of the project planning phase is critical to the success of simulation programs. In addition to planning and implementation, attention must be paid to ensuring sustainability.

The US spends more on healthcare than any other country but does not have better health outcomes than countries that spend far less [27]. Utilizing cost-effective programs that address this issue is imperative. Unit-based simulation programs in high-risk areas of medicine like obstetrics, is a way to increase the value proposition in the medical industry.

\section{Acknowledgements}

Thank you also to Wendy Wilcox for providing a supportive professional environment, Michele Follen for editorial assistance and humor, Liat Applewhite for exercising her passion, and Daisy Grueso for her partnership in change. Thank you to all the patients for whom I have cared and will care. They are critical to all endeavors. Thank you to my parents who have been key to my success.

\section{Conflicts of Interest}

The author declares no conflicts of interest regarding the publication of this paper.

\section{References}

[1] New York City Department of Health and Mental Hygiene (2016) Severe Maternal Morbidity in New York City, 2008-2012. New York.

[2] Institute of Medicine (2000) To Err Is Human: Building a Safer Health System. The National Academies Press, Washington DC. https://doi.org/10.17226/9728

[3] Motola, I., Devine, L.A., Chung, H.S., Sullivan, J.E. and Issenberg, S.B. (2013) Simulation in Healthcare Education: A Best Evidence Practical Guide. AMEE Guide No. 82, Medical Teacher, 35, e1511-e1530. https://doi.org/10.3109/0142159X.2013.818632

[4] Mitchell, P., Wynia, M., Golden, R., McNellis, B., Okun, S., Webb, C.E., Kohorn, V., 
et al. (2012) Core Principles \& Values of Effective Team-Based Care. Discussion Paper, Institute of Medicine, Washington DC.

https://nam.edu/perspectives-2012-core-principles-values-of-effective-team-basedhealth-care/ https://doi.org/10.31478/201210c

[5] Passiment, M., Sacks, H. and Huang G. (2011) Medical Simulation in Medical Education: Results of an AAMC Survey. The Association of American Medicine, Chicago. http://www.aamc.org

[6] (n.d.) Pilot at Zero Altitude: The History of Flight Simulators. https://www.proflight.com

[7] Georges, K. (2018) Simulation. The Lorraine Tregde Patient Safety Conference, New York.

[8] Shields, L.E., Goffman, D. and Caughey, A.B. (2017) Practice Bulletin No. 183: Postpartum Hemorrhage. Obstetrics and Gynecology, 130, 168-186. https://doi.org/10.1097/AOG.0000000000002346

[9] Papazian, J. and Kacmar, R.M. (2017) Obstetric Hemorrhage; Prevention, Recognition, and Treatment. Advances in Anesthesia, 35, 65-93.

https://doi.org/10.1016/j.aan.2017.07.004

[10] Developing and Sustaining High-Performance Work Teams (2015). https://www.shrm.org/resourcesandtools/tools-and-samples/tool/kits/pages/develo ping andsustatininghigh-performanceworkteams.aspx

[11] New York State Taskforce Maternal Mortality and Disparate Racial Outcomes (2019) Recommendations to the Governor to Reduce Maternal Mortality and Racial Disparitie. https://www.governor.ny.gov

[12] Nelissen, E., Ersdel, H., Mduma, E., Evjen-Olse, B., Twisk, J., Broerse, J., Roosmalen, J. and Stekelenburg, J. (2017) Clinical Performance and Patient Outcome after Simulation-Based Training in Prevention and Management of Postpartum Hemorrhage: An Educational Intervention Study in a Low-Resource Setting. BMC Pregnancy and Childbirth, 17, 1-9. https://doi.org/10.1186/s12884-017-1481-7

[13] Dahlberg, J., Nelson, M., Dahlgren, M.A. and Blomberg, M. (2018) Ten Years of Simulation Based Shoulder Dystocia Training Impact on Obstetric Outcome, Clinical Management, Staff Confidence, and the Pedagogical Practice-A Time Series Study. BMC Pregnancy and Childbirth, 18, 361. https://doi.org/10.1186/s12884-018-2001-0

[14] Al-Kadri, H.M., Dahwali, H., Al Airan, M., Elsherif, E., Tawfeeq, N., Mokhele, Y. and Brown, D. (2014) Postpartum Blood Loss Estimation. BMC Pregnancy and Childbirth, 14, Article No. 110. http://www.biomedcentral.com/1471-2393/14/110

[15] Fritz, J., Walker, D.M., Cohen, S., Angeles, G. and Lamadrid-Figueroa, H. (2017) Can a Simulation-Based Training Program Impact the Use of Evidence Based Routine Practices at Birth? Results of a Hospital-Based Cluster Randomized Trial in Mexico. PLoS ONE, 12, e0172623. https://doi.org/10.1371/journal.pone.0172623

[16] Mittal, V.K. (2015) Simulation Training-A Necessity for Future Surgeons. Indian Journal of Surgery, 77, 258-259. https://doi.org/10.1007/s12262-015-1396-3

[17] Bukari, H., Andreatta, P., Goldiez, B. and Rabelo, L. (2017) A Framework for Determining the Return on Investment of Simulation-Based Training in Health Care. The Journal of Health Care Organization, Provision, and Financing, 54, 0046958016687176. https://doi.org/10.1177/0046958016687176

[18] Blau, R. (2018) City Doled Out \$123 Million to Pay for Medical Malpractice Claims 
at Its Public Hospitals. Daily News.

https://www.nydailynews.com/new-york/ny-metro-hhc-medmal-20180920-story.html

[19] Robbennolt, J.K. (2009) Apologies and Medical Error. Clinical Orthopaedics and Related Research, 467, 376-382. https://doi.org/10.1007/s11999-008-0580-1

[20] Dunford, B.B., Boss, R.W., Boss, A.D., Shipp, A.J. and Angermeier, I. (2012) Is Burnout Static Or Dynamic? A Career Transition Perspective of Employee Burnout Trajectories. Journal of Applied Psychology, 3, 637-650. https://doi.org/10.1037/a0027060

[21] Weiner, B. (2009) A Theory of Organizational Readiness for Change. Implementation Science, 4, 67. https://www.ncbi.nlm.nih.gov/pmc/articles/PMC2770024 https://doi.org/10.1186/1748-5908-4-67

[22] (2009) Conducting Root Cause Analysis: A Resource Guide for Health Care Providers. http://www.gnyha.org

[23] (2012) Harvard Business Review Guide to Project Management: Motivate Your Team Avoid the Creep Deliver Results. Harvard Business School Publishing Corporation, Boston.

[24] Anderson, D.L. (2017) Organizational Development; the Process of Leading Organizational Change. 4th Edition, SAGE Publications, Thousand Oak.

[25] McChesney, C., Covey, S. and Huling, J. (2012) The 4 Disciplines of Execution. Simon \& Schuster UK Ltd., London.

[26] Banga, F.R., Truijens, S.E., Fransen, A.F., Dieleman, P.J., Heimel, P.J. and Oei, G.S. (2014) The Impact of Transmural Multiprofessional Simulation-Based Obstetric Team Training on Perinatal Outcome and Quality of Care in the Netherlands. BMC Medical Education, 14, 175. https://doi.org/10.1186/1472-6920-14-175

[27] Etehad, M. and Kim, K. (2017) The U.S. Spends More on Healthcare than Any Other Country But Not with Better Health Outcomes.

http://www.latimes.com/nation/la-na-healthcare-comparison-20170715-htmlstory. $\underline{\mathrm{html}}$

[28] Baldrige Performance Excellence Program (2019) 2019-2020 Baldrige Excellence Framework Healthcare: Proven Leadership and Management Practices for High Performance.

[29] Huynh, T.M., Baker, G.R., Bierman, A., Klein, D., Rudoler, D., Sharpe, G., Stukel, T., Tang, T., Wong, H.J. and Brown, A.D. (2014) Exploring Accountable Care in Canada Integrating Financial and Quality Incentives for Physicians and Hospitals. Final Report to the Ministry of Health and Long-Term Care and the Canadian Foundation for Healthcare Improvement.

https://www.cfhi-fcass.ca/sf-docs/default-source/reports/exploring-accountable-car e-brown-en.pdf

[30] Monod, C., Voekt, C.A., Gisin, M., Gisin, S. and Hoesli, I.M. (2014) Optimization of Competency in Obstetrical Emergencies: A Role for Simulation Training. Archives of Gynecology and Obstetrics, 289, 733-738. https://doi.org/10.1007/s00404-013-3111-6

[31] Thomas, R.K. (2015) Marketing Health Services. 3rd Edition, Health Administration Press, Chicago.

[32] Zuckerman, A.M. (2014) Healthcare Strategic Planning. 3rd Edition, Health Administration Press, Chicago.

[33] Dingley, C., Daugherty, D.K., Derieg, M.K. and Persing, R. (2008) Improving Patient Safety through Provider Communication Strategy Enhancements. In: Henrik- 
sen, K., Battles, J.B., Keyes, M.A. and Grady, M.L., Eds., Advances in Patient Safety: New Directions and Alternative Approaches, Vol. 3: Performance and Tools, Agency for Healthcare Research and Quality, Rockville.

[34] Martin, G.P., Weaver, S., Currie, G., Finn, R. and McDonald (2012) Innovation Sustainability in Challenging Health-Care Contexts: Embedding Clinically Led Change in Routine Practice. Health Services Management Research, 25, 190-199. https://doi.org/10.1177/0951484812474246

[35] (2016) Unit-Based (In-Situ) Simulation Manual. http://www.nychealthandhospitals.org/simulationcenter

[36] Yarber, L., Brownson, C.A., Jacob, R.R., Baker, E.A., Jones, E., Baumann, C. and Brownson, R.C. (2015) Evaluating a Train-the-Trainer Approach for Improving Capacity for Evidence-Based Decision Making in Public Health. BioMed Central Health Services Research, 15, Article No. 547. https://doi.org/10.1186/s12913-015-1224-2

[37] Nestel, D., Bearman, M., Brooks, P., Campher, Freeman, K., Greenhill, J., Watson, M., et al. (2016) A National Training Program for Simulation Educators and Technicians: Evaluation Strategy and Outcomes. BioMed Central Medical Education, 16, 25. https://doi.org/10.1186/s12909-016-0548-x

[38] Cooper, S., Porter, J. and Peach, L. (2013) Measuring Situation Awareness in Emergency Settings: A Systematic Review of Tools and Outcomes. Open Access Emergency Medicine, 6, 1-7. https://doi.org/10.2147/OAEM.S53679

[39] Lighter, D.E. (2011) ADVANCED Performance Improvement in Health Care. Jones and Bartlett Publishing, Sudbury.

[40] Pronovost, P.J. (2013) Enhancing Physicians' Use of Clinical Guidelines. JAMA, 310, 2501-2502. https://doi.org/10.1001/jama.2013.281334 http://Jama.Jamanetwork.com

[41] Panella, M., et al. (2003) Reducing Clinical Variations with Clinical Pathways: Do Pathways Work? International Journal for Quality in Health Care, 15, 509-521. https://doi.org/10.1093/intqhc/mzg057

[42] Chou, M. (2006) Litigation in Obstetrics: A Lesson Learnt and a Lesson to Share. Taiwanese Journal of Obstetrics and Gynecology, 45, 1-9. https://doi.org/10.1016/S1028-4559(09)60183-2

[43] Prakash, B. (2010) Patient Satisfaction. Journal of Cutaneous and Aesthetic Surgery, 3, 151-155. https://doi.org/10.4103/0974-2077.74491 


\section{Appendix}

\section{Definition of Terms}

1) Severe maternal morbidity (SMM) - life-threatening complications during or immediately after delivery [35]

2) Near-miss-An SMM event that could have occurred but did not. For example, the wrong medication was drawn into the syringe, but the patient did not receive it.

3) Obstetric hemorrhage- "cumulative blood loss greater than or equal to $1000 \mathrm{ml}$ or blood loss accompanied by signs and symptoms of hypovolemia within 24 hours after the birth process" [8].

4) Shoulder dystocia-when delivery of the baby's shoulder is obstructed by the mother's pelvic bone. The baby's head delivers through the vagina, but the shoulders do not. This can harm or kill a baby.

5) Brachial-plexus injury-The nerve that can be injured when a baby experiences a shoulder dystocia at birth. However, there are some that believe this injury can occur during a normal delivery as well.

6) Intubation-placing a breathing tube into a patient's airway to ensure that they receive oxygen. 